\title{
Research Paper: A6-Step Approach to Gain Higher Quality Results From Organotypic Hippocampal Brain Slices in a Traumatic Brain Injury Model
}

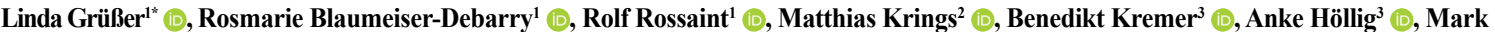 \\ Coburn ${ }^{1}$ (i)
}

1. Department of Anesthesiology, RWTH Aachen University Hospital, Aachen, Germany.

2 Department of Anesthesiology and Intensive Care, Medizinisches Zentrum StaedteRegion Aachen, Aachen, Germany.

3. Department of Neurosurgery, RWTH Aachen University Hospital, Aachen, Germany.

\begin{tabular}{l|l}
$\begin{array}{c}\text { use vour device to scan } \\
\text { and read the article online }\end{array}$ \\
$\begin{array}{l}\text { Cftation: Grüßer, L., Blaumeiser-Debarry, R., Rossaint, R., Krings, M., Kremer, B., \& Höllig, A. (2019). A 6-step Approach to } \\
\text { Gain Higher Quality Results From Organotypic Hippocampal Brain Slices in a Traumatic Brain Injury Model. Basic and Clinical } \\
\text { Neuroscience, 10(5), 485-498. http://dx.doi.org/10.32598/bcn.9.10.235 }\end{array}$ \\
doi http://dx.doi.org/10.32598/bcn.9.10.235
\end{tabular}

\section{(i) (3)}

Article info:

Received: 07 Sep 2018

First Revision: 27 Sep 2018

Accepted: 22 Mar 2019

Available Online: 01 Sep 2019

Keywords:

Organotypic hippocampal brain slices, In vitro model, Traumatic Brain Injury (TBI), Propidium Iodide (PI), Eligibility, Framework

\begin{abstract}
A B S T RA C T
Introduction: Organotypic Hippocampal Brain Slices (OHBS) provide an advantageous alternative to in vivo models to scrutinize Traumatic Brain Injury (TBI). We followed a wellestablished TBI protocol, but noticed that several factors may influence the results in such a setup. Here, we describe a structured approach to generate more comparable results and discuss why specific eligibility criteria should be applied.

Methods: We defined necessary checkpoints and developed inclusion and exclusion criteria that take the observed variation in such a model into consideration. Objective measures include the identification and exclusion of pre-damaged slices and outliers. Six steps were outlined in this study.

Results: A six-step approach to enhance comparability is proposed and summarized in a flowchart. We applied the suggested measures to data derived from our TBI-experiments examining the impact of three different interventions in 1459 OHBS. Our exemplary results show that through equal requirements set for all slices more precise findings are ensured.

Conclusion: Results in a TBI experiment on OHBS should be analyzed critically as inhomogeneities may occur. In order to ensure more precise findings, a structured approach of comparing the results should be followed. Further research is recommended to confirm and further develop this framework.
\end{abstract}




\section{Highlights}

- Several factors may influence the results in an organotypic hippocampal brain slice model scrutinizing traumatic brain injury.

- In order to enhance comparability, a structured approach comprising inclusion and exclusion criteria is outlined.

- The proposed approach is applied to data derived from a traumatic brain injury experiment and exemplary results show that through equal requirements set for all slices more precise findings are ensured.

\section{Plain Language Summary}

Several researchers utilized organotypic hippocampal slice (OHBS) cultures to scrutinize processes and possible treatment approaches for traumatic brain injury (TBI). Following a well-established protocol for a TBI-experiment we noticed that several factors may influence the results in such a set-up. We describe a structured approach to generate more precise results and discuss why specific eligibility criteria should be applied. We propose 6 steps comprising checkpoints, inclusion- and exclusion criteria in order to enhance comparability. The proposed approach is applied to data derived from our TBI experiment. The exemplary results demonstrate how the outcome can differ when a measure is applied vs. when it is not applied.

\section{Introduction}

rain cells have been studied in cultures for B over 100 years (Ross Granville Harrison, 1910; Rose G. Harrison, Greenman, Mall, \& Jackson, 1907; Hogue, 1952; Humpel, 2015; Millet \& Gillette, 2012). Over time, different methods were developed to preserve thin brain slices and keep them long-term viable (Cho, Wood, \& Bowlby, 2007; Gähwiler, 1981; Humpel, 2015; Stoppini, Buchs, \& Muller, 1991). Slice cultures can be prepared from a variety of brain regions; however, hippocampal slice cultures are used most frequently and replicate many aspects of the in vivo state, making investigations into mechanisms of brain synapses possible (Bahr et al., 1995; Cho et al., 2007; Finley et al., 2004; Gähwiler, Capogna, Debanne, McKinney, \& Thompson, 1997; Li, Han, \& Wang, 2016; Noraberg et al., 2005).

Given that the hippocampal slice set-up also provides decent experimental access and allows for detailed regulation of the extracellular environment, it is not surprising that these systems have been widely used to study neurogenesis or to investigate diseases such as Alzheimer's disease and stroke (Cho et al., 2007; Noraberg et al., 2005).

Several researchers utilized organotypic hippocampal slice cultures to scrutinize processes and possible treatment approaches for Traumatic Brain Injury (TBI) and different methods were described (Adamchik, Frantseva Weisspapir, Carlen, \& Perez Velazquez, 2000; Coburn, Maze, \& Franks, 2008; Di Pietro et al., 2010; Di Pietro et al., 2013; Harris et al., 2013; Hughes, Silva, Ahmed, Shreiber, \& Morrison, 2014; Krings, Höllig, Grüsser, Rossaint, \& Coburn, 2016; Loetscher et al., 2009; Miller et al., 2015; Morrison, Cater, Benham, \& Sundstrom, 2006; Morrison et al., 2003; Roehl et al., 2010; Rossaint et al., 2009; Schoeler et al., 2012; Smith et al., 2016; Vogel et al., 2016).

Following a well-established protocol for a TBI-experiment (as described in Methods), we noticed that several factors may influence the results in such a set-up (Adamchik et al., 2000; Coburn et al., 2008; Grüßer et al., 2017; Krings et al., 2016; Loetscher et al., 2009; Roehl et al., 2010; Rossaint et al., 2009; Schoeler et al., 2012). Here, we describe a structured approach to generate more comparable results and discuss why specific eligibility criteria should be applied. We propose a six-step approach of comparing our results to ensure more precise findings.

\section{Methods}

The data used for this study are derived from our TBIexperiments scrutinizing the impact of incubation with argon $50 \%$, desflurane $6 \%$, and their combination in 1459 OHBS. The final results of assessed interventions are presented and discussed in a separate paper (Grüßer et al., 2017). Here, we describe a specific approach to improve quality of results in such a set-up. The study 
was approved by the local Institutional Ethical Review Committee as well as the Institute of Animal Research Aachen University Hospital's animal protection representative according to the German animal protection law (TierSchG $\S 4$, III). First, we briefly describe the wellestablished TBI-protocol as described in the separate paper (Grüßer et al., 2017). Second, we outline the six steps developed to generate more comparable results (Grüßer et al., 2017).

\subsection{Organotypic hippocampal slices}

The OHBS were obtained from 5-7-day-old mice pups (C57BL/6N, Charles River Laboratories, Sulzfeld, Germany) and kept in cultures as summarized in Suppl. 1, as previously described (Coburn et al., 2008; Grüßer et al., 2017; Krings et al., 2016; Loetscher et al., 2009; Roehl et al., 2010; Rossaint et al., 2009; Schoeler et al., 2012; Stoppini et al., 1991).

\subsection{Traumatic brain injury}

The experiments were performed as described before (Grüßer et al., 2017); For the experiment the growth medium (GM) was exchanged by an experimental medium (EM) containing propium iodide (PI). Eventually, baseline fluorescence images, showing the cell damage before the experiment was started, were taken and tissue plates were randomly assigned to their groups.Our experiment included four trauma and four non-trauma groups in which no and three different kinds of atmospheric interventions took place.

When assigned to a group, the TBI was elicited in a similar way to the procedure described by other researchers (Adamchik et al., 2000; Coburn et al., 2008; Krings et al., 2016; Loetscher et al., 2009). A round stylus was positioned $7 \mathrm{~mm}$ above the hippocampus slice and was then dropped dropped onto the slice positioned at a marked spot. After TBI was performed, EM was exchanged once more, and slices were incubated in the respective atmosphere. When assigned to a non-TBI group, slices underwent the same procedure except that no trauma was induced. After an incubation period of two hours, final fluorescence imaging took place for all groups.

\subsection{Microscopy and assessment of cell death}

PI, a dyeing agent that rapidly enters cells with damaged membranes and binds to their DNA, was applied to assess the proportion of cell death (Macklis \& Madison, 1990). Images were captured with a fluorescence mi- croscope and MetaVue software (MetaVue, Molecular Devices, Sunnyvale, CA, USA). Using ImageJ software (National Institutes of Health, Bethesda, MD, USA), a histogram of the red channel was generated. The histogram listed the sum of all pixels sharing the same greyscale value from 0 to 255 . Previous studies have shown that values below a threshold of 100 are caused by background fluorescence so only values above this threshold were summed up to assess the extent of the cell injury (Loetscher et al., 2009; Roehl et al., 2010; Schoeler et al., 2012). Hence, the sum of the pixels in an image represents the damage of a slice, as demonstrated in the separate paper (Grüßer et al., 2017).

SPSS v. 22 (IBM SPSS Statistics, IBM Corporation, Somers, NY, USA), was used to calculate the Mean value \pm Standard Error of the mean of the sum of pixels for each group. As a reference value, the trauma/no intervention group's mean value was normalized to unity. Statistical relevance was determined with the help of the Student t-test. P-values $<0.05$ were considered to be significant.

2.4. Recommendation: Six steps to generate more comparable results

Through a closer investigation of OHBS in our TBIexperiment, we identified several factors that may influence the results. In order to enhance comparability, we developed the following six steps, which are summarized in a model as shown in Figure 1.

\subsubsection{Confirmation}

In a first step, it is recommended to verify that each picture taken shows only one slice, amounts to the pixels of this slice, and thus fully shows its cell damage. We suggest enhancing the contrast of each image captured (only for better visibility-pixel counts are determined as explained above) (Figure 2.1). This allows for the identification of a second slice in the picture and eventually the blackening of this area. Further, a thorough evaluation of each photograph is advantageous to detect small PI-clots or fusels. Finally, it can be ensured that each image taken before the experiment is matched with the correct one at later time-points of assessment (Figure 2.1, Example image step 1).

\subsubsection{Identification of pre-damaged slices at base-} line assessment and exclusion

Other researchers have pointed to the fact, that they excluded slices from their experiments if they were dam- 


\section{Confirmation}

Each picture taken shows only one slice, amounts to the pixels of this slice and thus represents its cell damage. Enhance contrast to identify fusels or PI-clots, to confirm that the correct slices at baseline assessment and after incubation time are paired and that only one slice is captured in one image.

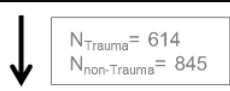

2. Identification of pre-damaged slices at baseline assessment and exclusion

If baseline image histograms already present with a number of pixels above a set threshold, they should be sorted out as they are considered to be pre-damaged.

\section{$\downarrow \begin{aligned} & N_{\text {trauma }}=393 \\ & N_{\text {non- }- \text { Trauma }}=568\end{aligned}$}

\section{Preparation margin errors}

Install a threshold: If PI-detectable cell death in a margin exceeds this threshold, this margin is considered to be prominent enough to be an error due to errors in preparation and its pixels are not added to the full integral of pixels in this picture. If it is smaller or equal to this threshold, the amount of pixels of the entire picture is taken into account.

$$
\downarrow \begin{aligned}
& N_{\text {Trauma }}=399 \\
& N_{\text {non-Truuma }}=637
\end{aligned}
$$

\section{Morphological peculiarities}

These peculiarities include what we describe as "holes", "frayed margins", "emigrated cells". The reason for these phenomena is unknown. Excluding all these slices would significantly decrease the number of slices available in specific groups. Further research is recommended. For now, we refer to the threshold "identification of pre-damaged slices at baseline assessment and exclusion".

\section{Variation of trauma intensities}

Define a scope of trauma strength to investigate and identify unrealistic outliers in the trauma groups. One way is to perform boxplot analysis in the positive control group and identify extreme outliers. The new mean value of the positive control group plus $2 x$ the standard deviation serves as an upper threshold for all the other trauma groups. Slices that were clearly not hit properly by the pin should be excluded. For future researchers we recommend to set a defined size prior to the experiment that has to be met to be considered for inclusion concerning the hippocampus itself, the pin's impression and the area of secondary injury.

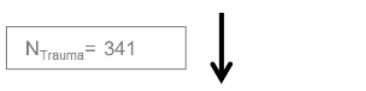

more precise results

6. Susceptibility of non-trauma groups Adjusting the focus of the camera when taking pictures is crucial in particular with regards to the non-trauma groups where trauma intensities are minimal. A useful and easy way to double-check your setting is, first, to exclude all slices that presented with lower number of pixels after incubation than at baseline assessment. Second, perform boxplot-analysis in each group to identify and exclude outliers. Thus, not only slices presenting with excessive trauma intensities because pictures showing the predamage at baseline assessment were blurry, but also slices damaged due to manual incautiousness or temperature change are excluded. For future researchers we recommend to set a defined size of the hippocampus prior to the experiment that has to be met to be considered for inclusion.

Figure 1: Flowchart summarizing the six-step approach

The flowchart summarizes the six-step approach; we applied the proposed model to the data derived from our experiments on 1459 OHBS, as mentioned above.

aged at baseline assessment or showed high levels of PI fluorescence (Adamchik et al., 2000; Harris et al., 2013; Krings et al., 2016; Miller et al., 2015). However, no details were described, and in many papers, we did not find any information that slices were excluded. As inserts are moved for medium changes and are exposed to temperature fluctuations, pre-damage to the slice at baseline assessment is a common feature. Moreover, preparation techniques and timing may still improve within the course of the experiment. 
It is crucial to avoid pre-damaged slices entering into the experiment, as their trauma intensity may falsify the results after the incubation time. Since it is unknown whether a higher amount of injured cells at baseline assessment might affect or even exponentially increase the development of secondary injury, only minimally pre-damaged slices should be considered within the experiment. In order to avoid bias in identification and outsourcing of these slices, an objective threshold should be defined. In our experiment, if baseline image histograms presented more than 1000 pixels, they would be sorted out as they were considered to be pre-damaged (Figure 2.2, Example image of step 2).

\subsubsection{Preparation margin error}

Motor skills develop over training sessions and probably through different stages (Luft \& Buitrago, 2005). Similarly, preparation techniques improve when regularly dissecting the hippocampi. A typical feature we observed, especially within the first rounds of prepared slices, was a prominent margin due to-as suggested here-errors in preparation. Even though preparation steps were always strictly followed, some of the slices presented with noticeable frames of PI-detectable cells. The pictures taken at baseline assessment did not necessarily exceed an integral of $>1000$ pixels. After an incubation period of two hours, however, PI fluorescence was highly visible, and thus slices with such a prominent margin distorted the outcome of the affected groups. In order to objectively decide on what was a margin due to an error in preparation and what was average damage which would inevitably occur, the following threshold was applied: if the PI-detectable cell death in a margin exceeded $>250$ pixels, this margin was considered to be prominent enough to be an error stemming from errors in the preparation and its pixels were not added to the full integral of pixels in this picture (Figure 2.3). We took the same approach for slices presenting with a "one-celled" margin, which was observed in only two experimental series (Figure 2.3, Example image of step 3).

\subsubsection{Morphological peculiarities}

Within the course of the study, we noticed several morphological peculiarities in varying degrees. These peculiarities include what we describe as "holes", "frayed margins", and "emigrated cells" (Figure 2.4). A closer investigation of these phenomena is suggested for future studies, as we do not know why they occurred and cannot evaluate whether electrophysiological consequences are entailed. We observed that "holes" often went in line with "frayed margins" and "emigrated cells". However, these features also occurred independently from one another. Additionally, by enhancing the contrast of the image, we noticed that emigrated cells also existed at baseline assessment, but this was not always PI-detectable by PI. Excluding all slices with a specific morphology would significantly decrease the number of slices available in specific groups. Therefore, we decided to refer to step 1: if slices with peculiarities presented with $<1000$ pixels at baseline assessment, they would be taken into consideration for the experiment (Figure 2.4, Example image of step 4).

\subsubsection{Variation of trauma intensities}

Within the course of the experiment, we observed that trauma intensities varied over time and that some experimental series, even within one group presented with different trauma severities inspite the same dropping height was ensured (Figure 2.5). Thus, we saw the necessity to define a scope of trauma strength. A boxplot analysis was performed for the trauma control group, and extreme outliers were identified $(\mathrm{n}=2)$ and excluded. The new mean value of the trauma control group plus two times the standard deviation served as an upper threshold for all the other trauma groups.

Concerning trauma intensities, it should also be taken into consideration that depending on where the pin hits the slice, more or less tissue can be injured by secondary injury, i.e., if the pin hits the slice close to the margin there is less hippocampus to be affected in the following time. Also - as we experienced - the shapes of the hippocampi vary where we found it is unlikely to work with extremely similar contoured slices. Hence, our approach was to take all slices into consideration except for those that were clearly not hit properly by the pin $(n=7)$.

In our experiment, we identified several borderline cases, mostly in the intervention group presenting with the least secondary injury, where it appeared to be difficult to make a clear decision. For future researchers we would recommend to set a defined size before the experiment that has to be met to be considered for inclusion - concerning the hippocampus itself (which is also of importance for the non-trauma groups), the pin's impression and the area of secondary injury (Figure 2.5, Example image for step 5).

\subsubsection{Susceptibility of non-trauma groups}

We noticed that it is crucial to adjust the focus of the camera when taking pictures, particularly in regards to the non-trauma groups, where trauma intensities are 

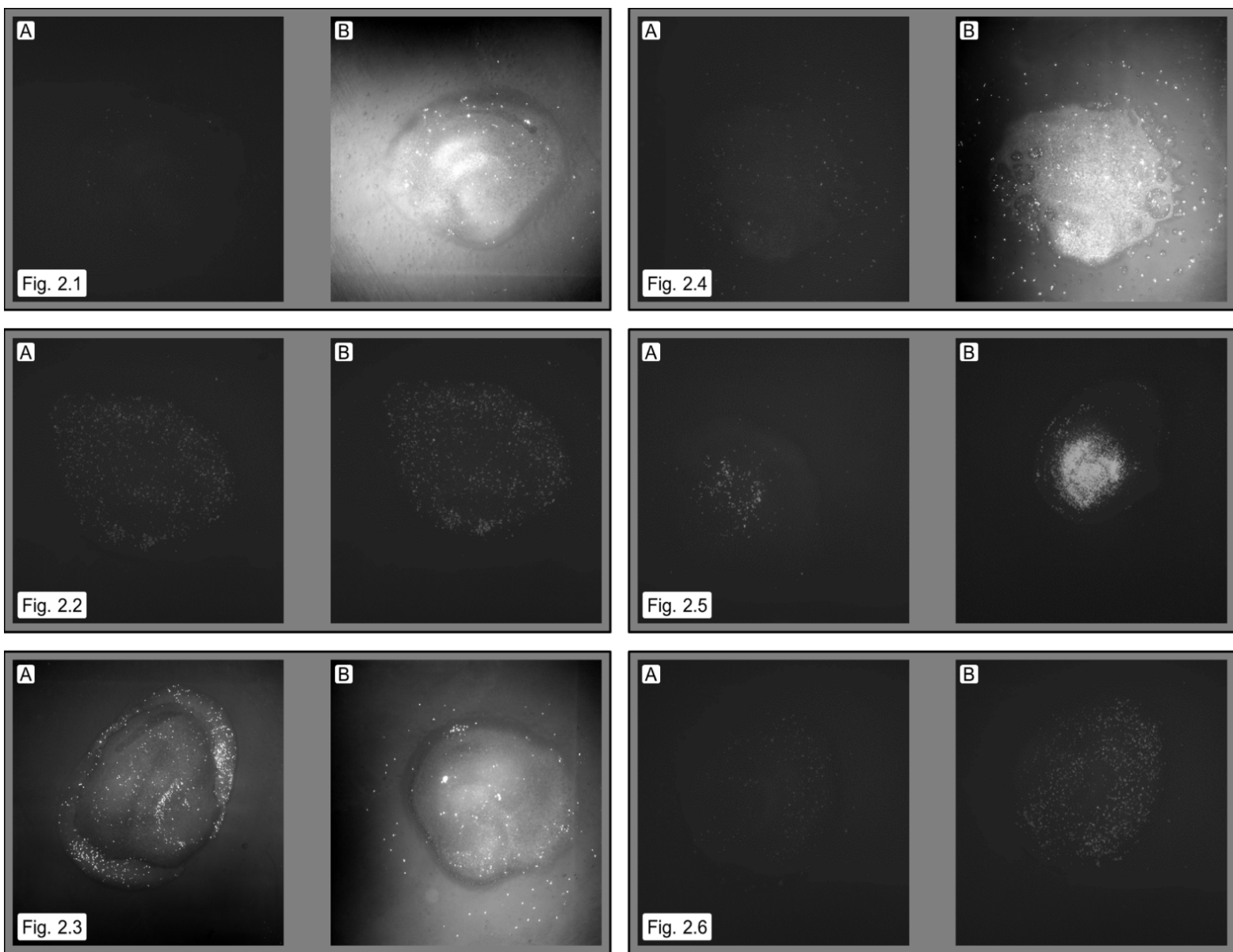

Figure 2. Example images of steps

NEUR SCIENCE

2.1: Example image of step 1: This picture is taken at the baseline assessment. A: Shows the image generated by ImageJ software via the red channel. B: shows the same slice after having enhanced the contrast; Figure 2.2: Example image of step 2: Picture A shows a slice at baseline assessment. Due the threshold number of pixels at baseline assessment $<1000$, this pre-damaged slice was restrained from entering the experiment. Thus, it could be avoided that the picture showing the trauma intensity after the incubation period (B) distorted the final results (B); Figure 2.3 Example image of step 3: Picture A shows a margin, which is probably due to errors within the preparation process. Picture B shows damage which probably occurs inevitably; Figure 2.4: Example image of step 4: Morphological peculiarities include what we called "holes", "frayed margin", and "emigrated cells". These peculiarities are often only seen when the contrast is enhanced (A: picture generated by ImageJ; B: contrast additionally enhanced). The slice in the image (picture taken after the incubation time) shows all three peculiarities. The reason for these phenomena are unknown; Figure 2.5: Example image of step 5: As trauma intensities vary, it is important to set a scope of trauma strength. Picture A is an example of a very weak trauma, and picture B shows one of the slices excluded by the established threshold; Figure 2.6: Example image of step 6: Picture A shows a slice at baseline assessment, which was not excluded by the threshold \#pixels at baseline assessment $<1000$. Picture B shows the same slice after the incubation period. Through the performed boxplot analysis, this slice was identified and excluded eventually.

minimal. Blurry images can present with a lower amount of greyscale pixels as the majority of pixels are evaluated as background fluorescence. Hence, in a few cases, it might still be possible that pre-damaged slices are not excluded by the aforementioned 1000-pixels threshold and then present with excessive greyscale values after the incubation time. Also- the other way round- a misleadingly low number of pixels is possible when the picture was taken after the incubation time is blurry.
In order to prevent biases by manually evaluating the sharpness of hundreds of pictures, we first excluded all images in the non-trauma groups that had lower pixel numbers after the incubation period than at baseline assessment. We subjectively excluded 11 slices due to their size as, for example, they were too small to be considered a hippocampal slice. As mentioned above, in the future, a defined size of the hippocampus should be set prior to the experiment. In a next step, we performed a boxplot-analysis in each non-trauma group to exclude outliers. Thus, not only slices presenting with excessive 

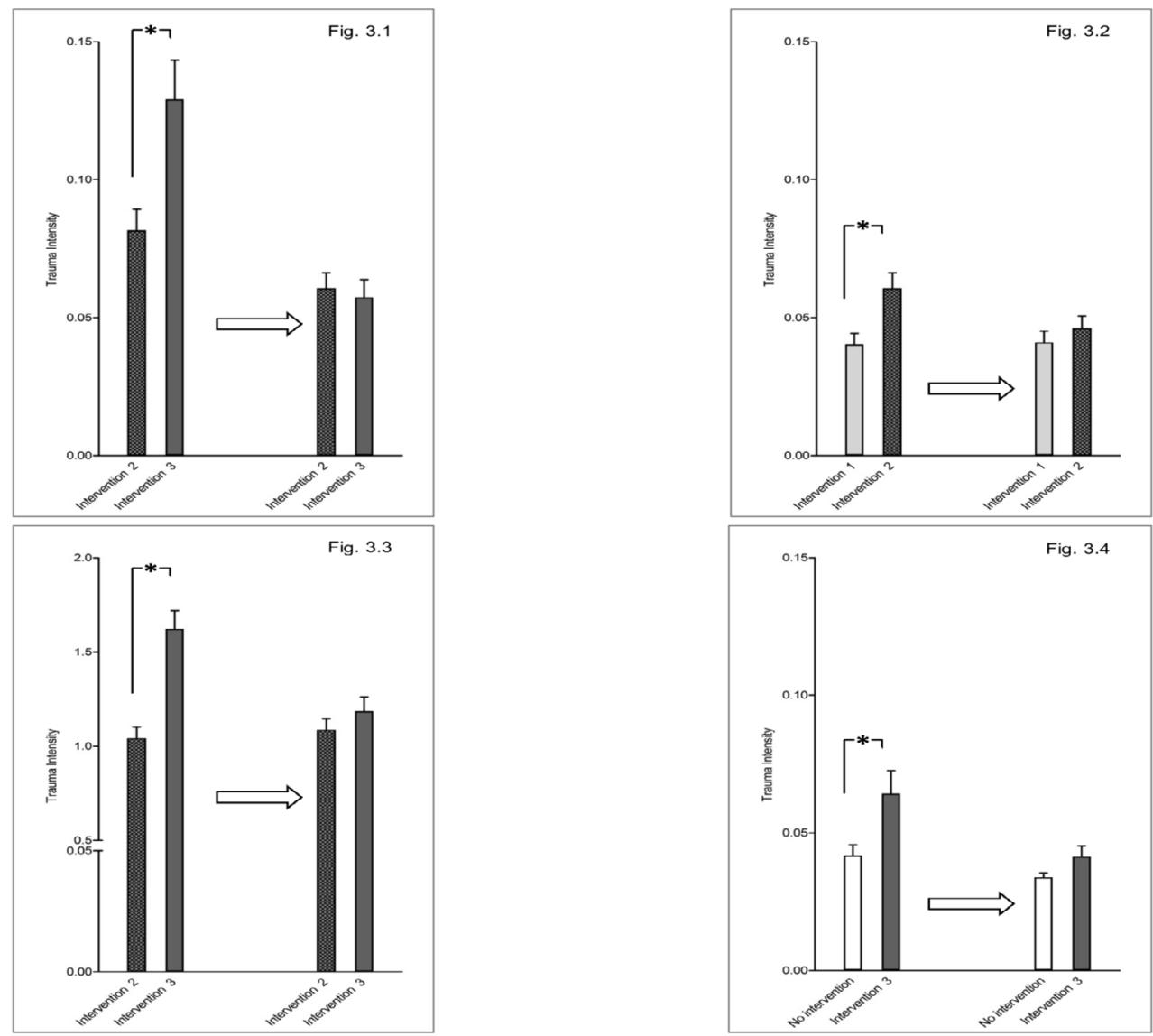

Figure 3. Results demonstrate that more precise findings are ascertained

NEUR SCIENCE

Figure 3.1: This image shows the trauma intensity of two non-trauma groups without applying a threshold concerning predamage compared to when a threshold is used (step 2). The difference in between the two groups is not significant anymore when the threshold concerning pre-damage is applied ( $\mathrm{P}=0.004$ vs. $\mathrm{P}=0.716)$; Figure 3.2: This image shows the results of two non-trauma groups before and after taking margins possibly caused by errors in the preparatory process into consideration. Our established threshold (step 3) aims to identify those errors and subtract the cell death of those margin areas. The difference in between the two groups is not significant when those margins are considered ( $\mathrm{P}=0.004$ vs. $\mathrm{P}=0.444)$; Figure 3.3: This image shows that the difference in between the two trauma groups is not significant when outliers are identified and excluded, as described in step 5. The difference in between the two groups seems to be significant at first glance, but it is not ( $\mathrm{P}<0.001$ vs. $\mathrm{P}=0.304)$; Figure 3.4: This image shows This image shows that also in the non-trauma groups results can differ when outliers are identified and excluded (step 6). The results show that the difference between the two groups is not as striking as expected at first $(\mathrm{P}=0.016$ vs. $\mathrm{P}=0.075)$.

trauma intensities due to pictures showing the pre-damage at baseline assessment were blurry, but also slices damaged due to manual incautiousness or temperature change were excluded in an objective manner (Figure 2-6, Example image of step 6).

\section{Results}

A six step approach to enhance comparability is proposed. We applied the proposed model to the data derived from our experiments on 1459 OHBS as mentioned above (Figure 1). In the following we present exemplary results that demonstrate how the outcome can differ when a measure is applied vs. when it is not applied.
After the first checkpoint, step 1, (confirmation), 1459 slices were assessed for pre-damage, step 2. As Figure 3.1 shows, the difference between two non-trauma groups seems to be significant at first glance; yet, when it is ascertained that baseline slices in both groups were presented with similar PI-detectable trauma intensities, this difference did not turn out to be significant anymore $(\mathrm{P}=0.004$ vs. $\mathrm{P}=0.716$. In a next step, the slices were screened for preparatory margins (step 3). Figure 3.2 demonstrates the difference in results when preparation errors are not vs. when they are taken into consideration. Misleadingly, the non-trauma/intervention group 2 would have been considered significantly more injured 
than the non-trauma/intervention group $1(\mathrm{P}=0.004 \mathrm{vs}$. $\mathrm{P}=0.444$

Without having applied this measure, a difference in between the two groups would have mistakenly been ascribed to the specific interventions. By subtracting margins stemming from errors in the preparatory process, more slices met the baseline assessment threshold and were considered for the next steps (393 slices after step 2 vs. 399 slices after step 3 in the trauma groups and 568 vs. 637 slices in the non-trauma groups). Morphological peculiarities, as described in step 4 require further investigation.

Step 5 aims to set a scope concerning trauma intensities in the trauma groups. Figure 3.3 demonstrates that without a set scope, the trauma/intervention group 3 would misleadingly have been considered to generate a neuroharming effect in comparison to the trauma/intervention group 2 ( $\mathrm{P}<0.001$ vs. $\mathrm{P}=0.304$. Identifying and excluding outliers in the non-trauma groups (step 6) revealed that the difference between the non-trauma/no intervention group and the non-trauma/intervention group 3 was not significant $(\mathrm{P}=0.016$ vs. $\mathrm{P}=0.075$ (Figure 3.4 ).

Within the six-step approach the number of slices was narrowed down to 547 pictures in the non-trauma groups and 341 pictures in the trauma groups (Figure 1). Thus, $61 \%$ of the slices met the proposed inclusion criteria (Figure 3; results demonstrating more precise findings).

\section{Discussion}

Working with OHBS in a TBI-experiment, we noticed that several factors may influence the results. To our knowledge, detailed objective manners to generate more comparable results in such a susceptible set-up have not been discussed so far. The proposed model aims to set clear requirements in order to diminish the possibility of pre-damaged slices entering into the experiment and outliers skewing the final results. This methodological approach provides a first framework to cope with the broad variation observed in these models. Our exemplary results show that through equal requirements set for all slices more precise findings are ensured.

In vitro models provide a useful tool to scrutinize processes and treatment options in the initial step. In between dissociated cell cultures and the in vivo state, OHBS present a potent alternative (Humpel, 2015; Li et al., 2016; Morrison, Saatman, Meaney, \& McIntosh, 1998; Noraberg et al., 2005). The hippocampal circuits in organotypic brain slices usually derived from early postnatal animals, are found to be indeed similar to the in vivo state but some differences have been detected (Gähwiler et al., 1997; Gogolla, Galimberti, DePaola, \& Caroni, 2006).

Of course, variables like perfusion, intracranial pressure, or the blood-brain barrier are not yet reflected in organotypic brain slice models, and the use of adult donor animals for organotypic brain slices has been scarce (Humpel, 2015; Li et al., 2016). Nonetheless, slices have been shown to remain $100-150 \mu \mathrm{m}$ thick with a threedimensional organization of connectivity (Gähwiler et al., 1997; Gogolla et al., 2006; Li et al., 2016; Stoppini et al., 1991). Notably, outcomes of studies working with OHBS have successfully been reproduced in in vivo models demonstrating the potential of this setup. For example, argon and xenon displayed a neuroprotective profile in OHBS models which could be substantiated in in vivo studies (Brücken et al., 2013; Coburn et al., 2008; Harris et al., 2013; Loetscher et al., 2009; Zhuang et al., 2012).

An important implication of research with organotypic brain slices is the reduction of the number of animal experiments (Humpel, 2015; Li et al., 2016). Further, in in vitro models of CNS injury, the associated costs are lower (Morrison et al., 1998). In their reviews on brain slice models, Noraberg et al. concluded that organotypic brain slices emerged to be an increasingly popular tool to investigate neurological diseases and Cho et al. assert that through advancements of brain slices and technological innovations in this field there is now a tremendous potential to address questions quicker (Cho et al., 2007; Noraberg et al., 2005).

To our knowledge, clear and objective procedures to make results more comparable within one experiment and among different studies have not been discussed in detail yet. Retrospectively, Guy et al.'s method of determining slice thickness as a parameter for OHBS health might present a useful additional measure (Guy, Rupert, Sandberg, \& Weber, 2011). In their review, Humpel et al. generally recommend to withdraw thick and not flattened slices. The outgrowth of cells from the edge of the living slices and a change in color to transparent grey color in the first week are criteria described for 'good' slices (Humpel, 2015).

De Simoni et al. point to the fact that it is difficult to identify pyramidal cell layers in an unhealthy slice (De Simoni \& Yu, 2006). Also, Gogolla et al.'s recommendation not to culture slices with a lesioned CA1 region or a lesioned or a detached dentate gyrus but only slices 
'with nice cell layers in the dentate gyrus and CA1-3 and smooth margins' seems to be a reasonable approach to be taken into consideration for future researchers (Gogolla et al., 2006). Our model mainly relied on PI-detectable cell death, however other cell-specific markers than PI can be used (Humpel, 2015).

Experimental papers often do not report about slice selection. Some researchers have pointed to difficulties, e.g., variation in PI-uptake, incomplete layers or predamage, but did not explain objective criteria concerning how or why brain slices were excluded or included (Adamchik et al., 2000; Harris et al., 2013; Krings et al., 2016; Li et al., 2016; Miller et al., 2015). For clinical trials, participants must meet specific standards, they must fulfil 'inclusion criteria' or are prohibited from participating due to 'exclusion criteria' (National Institutes of Health, 2016). This does not only protect the participants' safety but also ensures that the subsequent results are more comparable as the study population is more alike.

Within our TBI-experiment in OHBS, we found that results differ when hippocampal brain slices undergo specific assessments for eligibility eligibility. We found that hippocampal brain slices can present with predamage or specific peculiarities and identified factors that might affect the final outcome.

In general, researchers should aim to work as cautiously as possible to avoid imprecise preparation, long temperature changes, accidental shaking, or changes in the experimental process. Albeit all precautions are taken, organizational challenges with regards to timing, the microscope setting, or the medium may occur. Fluctuations in the severity of trauma may distort the outcome. Even though the reasons are not clear, our results show that hippocampal brain slices do not necessarily present an unconditionally homogenous group. Thus, eligibility criteria for OHBS present a plausible conclusion.

In order to avoid bias by manually evaluating slices, the criteria set should be as objective as possible. If slices are very uniform and interventions are very consistent, the eligibility criteria do not interfere with the final outcome. If any inconsistencies occur, the applied measures increase the chances of more precise conclusions to be drawn. In our experiment, for example, a considerable amount of data were deemed unusable as the slices were considered to be pre-damaged. Even though this threshold was chosen arbitrarily, we could thus ensure that equal requirements were set for all experimental series.
Our results demonstrate that outcomes in a TBI experiment with OHBS should be analyzed critically as inhomogeneities may occur. Altogether, the proposed six steps are designed to take possible errors into consideration, help to contextualize the findings and make results more comparable. In the future, enhanced comparability may decrease the number of animals needed.

\section{Limitations}

This framework presents a first attempt to enhance comparability in an in vitro model of TBI in OHBS. Further research is recommended to confirm and further develop this model in studies designed for this cause. It may improve quality of the results if the general recommendations listed above were specified and also considered in the proposed approach as PI-detectable cell death and the proposed measures might not be sufficient.

Some challenges remain that could not be adequately addressed. First, the proposed thresholds are chosen arbitrarily, and future researchers are recommended to find a statistical solution of what should be considered ineligible, i.e. pre-damage.

Another disadvantage might be that obvious preparatory margins with less than 250 pixels could still have entered the experiment. As the margins had to be identified and blackened manually, this represents a subjective component and remains a potential source of error. We aimed to blacken only areas of cell death, possibly stemming from errors in the preparation process as the morphological peculiarities and their origin are unknown. However, it was not always clearly distinguishable if a specific area should be considered as a "preparatory error", a "frayed margin", a "hole", or as "emigrated cells". For further studies, we would also recommend to constantly allocate slices prepared in one series to all groups in order to distribute potential errors more equally.

Despite all measures taken, slices e.g., too small to be considered as a hippocampus or slices that were not clearly hit by the pin had to be identified. We tried to minimize the number of slices excluded manually, so this presents a subjective choice, and we recommend defining inclusion criteria concerning the size of the hippocampi and the trauma area prior to the experiment for future researchers. This seems particularly useful as the trauma produced by the pin in our experiment does not always resemble a proper circle, which can also be seen in images shown in other papers (Adamchik et al., 2000; Roehl et al., 2010). It is unclear whether this is typical as the injury spreads non-circularly throughout the slice 
depending on differences in susceptibility of cells or is caused by other factors, as, e.g., an oblique fall.

Although the framework was constructed to avoid future errors, there may be cases that were not caught. For example, our measures taken to exclude the most blurry pictures might not be sufficient enough to identify slightly blurry pictures. We included all slices and applied the measures proposed; however, numerical thresholds cannot identify, i.e., morphological completeness. Notwithstanding that a considerable amount of data is sorted out when the inclusion criteria are not met, this model presents the first approach to make research with hippocampal brain slices in an in vitro model of TBI more comparable.

\section{Conclusion}

Several factors may influence experimental outcomes in a TBI-experiment in OHBS. In order to reach the most precise outcome, checkpoints should be set, predamaged slices have to be defined and restrained from entering into the experiment, and unrealistic outliers have to be detected and eventually excluded. Objective approaches presented in our framework include the installation of thresholds and boxplot-analyses. Our exemplary results demonstrate that more comparable findings can thus be ensured. Further research is recommended to confirm and further develop this model and to scrutinize morphological peculiarities, their origin and their potential impact on cell survival and electrophysiological functioning.

\section{Ethical Considerations}

\section{Compliance with ethical guidelines}

All ethical principles were considered in this article.

\section{Funding}

This research did not receive any specific grant from funding agencies in the public, commercial, or not-forprofit sectors.

\section{Authors' contributions}

All authors equally contributed in preparing this article.

\section{Conflict of interest}

The authors declared no conflict of interest.

\section{Acknowledgments}

We thank Prof. Joachim Weis, head of the Department of Neuropathology, RWTH Aachen University Hospital, Aachen, Germany, and his team for their support in the culturing of the slices.

\section{References}

Adamchik, Y., Frantseva, M. V., Weisspapir, M., Carlen, P. L., \& Perez Velazquez, J. L. (2000). Methods to induce primary and secondary traumatic damage in organotypic hippocampal slice cultures. Brain Research Protocols, 5(2), 153-8. [DOI:10.1016/S1385-299X(00)00007-6]

Bahr, B. A., Kessler, M., Rivera, S., Vanderklish, P. W., Hall, R A., Mutneja, M. S., et al. (1995). Stable maintenance of glutamate receptors and other synaptic components in long-term hippocampal slices. Hippocampus, 5(5), 425-39. [DOI:10.1002/ hipo.450050505] [PMID]

Brücken, A., Cizen, A., Fera, C., Meinhardt, A., Weis, J., Nolte, K., et al. (2013). Argon reduces neurohistopathological damage and preserves functional recovery after cardiac arrest in rats. British Journal of Anaesthesia, 110(suppl. 1), i106-i12. [DOI:10.1093/bja/aes509] [PMID]

Cho, S., Wood, A., \& Bowlby, M. R. (2007). Brain slices as models for neurodegenerative disease and screening platforms to identify novel therapeutics. Current Neuropharmacology, 5(1) 19-33. [DOI:10.2174/157015907780077105] [PMID] [PMCID]

Coburn, M., Maze, M., \& Franks, N. P. (2008). The neuroprotective effects of xenon and helium in an in vitro model of traumatic brain injury. Critical Care Medicine, 36(2), 588-95. [DOI:10.1097/01.CCM.0B013E3181611F8A6] [PMID]

De Simoni, A., Yu, L. M. (2006). Preparation of organotypic hippocampal slice cultures: Interface method. Nature Protocols, 1(3), 1439-45. [DOI:10.1038/nprot.2006.228] [PMID]

Di Pietro, V., Amin, D., Pernagallo, S., Lazzarino, G., Tavazzi, B., Vagnozzi, R., et al. (2010). Transcriptomics of traumatic brain injury: gene expression and molecular pathways of different grades of insult in a rat organotypic hippocampal culture model. Journal of Neurotrauma, 27(2), 349-59. [DOI:10.1089/ neu.2009.1095] [PMID]

Di Pietro, V., Amorini, A. M., Tavazzi, B., Hovda, D. A., Signoretti, S., Giza, C. C., et al. (2013). Potentially neuroprotective gene modulation in an in vitro model of mild traumatic brain injury. Molecular and Cellular Biochemistry, 375(1-2), 185-98 [DOI:10.1007/s11010-012-1541-2] [PMID]

Finley, M., Fairman, D., Liu, D., Li, P., Wood, A., \& Cho, S (2004). Functional validation of adult hippocampal organotypic cultures as an in vitro model of brain injury. Brain Research, 1001(1-2), 125-32. [DOI:10.1016/j.brainres.2003.12.009] [PMID]

Gähwiler, B. H. (1981). Organotypic monolayer cultures of nervous tissue. Journal of Neuroscience Methods, 4(4), 329-42. [DOI:10.1016/0165-0270(81)90003-0] 
Gähwiler, B. H., Capogna, M., Debanne, D., McKinney, R. A., \& Thompson, S. M. (1997). Organotypic slice cultures: A technique has come of age. Trends in Neurosciences, 20(10), 471-7. [DOI:10.1016/S0166-2236(97)01122-3]

Gogolla, N., Galimberti, I., DePaola, V., \& Caroni, P. (2006) Preparation of organotypic hippocampal slice cultures for long-term live imaging. Nature Protocols, 1(3), 1165-71. [DOI:10.1038/nprot.2006.168] [PMID]

Grüßer, L., Blaumeiser-Debarry, R., Krings, M., Kremer, B., Höllig, A., Rossaint, R., et al. (2017). Argon attenuates the emergence of secondary injury after traumatic brain injury within a 2-hour incubation period compared to desflurane: An in vitro study. Medical Gas Research, 7(2), 93-100. [DOI:10.4103/20459912.208512] [PMID] [PMCID]

Guy, Y., Rupert, A. E., Sandberg, M., \& Weber, S. G. (2011). A simple method for measuring organotypic tissue slice culture thickness. Journal of Neuroscience Methods, 199(1), 78-81. [DOI:10.1016/j.jneumeth.2011.03.027] [PMID] [PMCID]

Harris, K., Armstrong, S. P., Campos-Pires, R., Kiru, L., Franks, N. P., \& Dickinson, R. (2013). Neuroprotection against traumatic brain injury by xenon, but not argon, is mediated by inhibition at the N-methyl-D-aspartate receptor glycine site. Anesthesiology, 119(5), 1137-48. [DOI:10.1097/ ALN.0b013e3182a2a265] [PMID]

Harrison, R. G. (1910). The outgrowth of the nerve fiber as a mode of protoplasmic movement. Journal of Experimental Zoology, 9(4), 787-846. [DOI:10.1002/jez.1400090405]

Harrison, R. G., Greenman, M. J., Mall, F. P., \& Jackson, C. M. (1907). Observations of the living developing nerve fiber. The Anatomical Record, 1(5), 116-28. [DOI:10.1002/ar.1090010503]

Hogue, M. J. (1952). Review of studies of human fetal brain cells in tissue cultures. Etudes Neonatales, 1(2), 1-13. [DOI:10.1016/0014-4827(52)90129-8]

Hughes, R. H., Silva, V. A., Ahmed, I., Shreiber, D. I., \& Morrison III, B. (2014). Neuroprotection by genipin against reactive oxygen and reactive nitrogen species-mediated injury in organotypic hippocampal slice cultures. Brain Research, 1543, 308-14. [DOI:10.1016/j.brainres.2013.11.020] [PMID]

Humpel, C. (2015). Organotypic brain slice cultures: A review. Neuroscience, 305, 86-98. [DOI:10.1016/j.neuroscience.2015.07.086] [PMID] [PMCID]

Krings, M., Höllig, A., Grüsser, L., Rossaint, R., \& Coburn, M. (2016). Desflurane impairs outcome of organotypic hippocampal slices in an in vitro model of traumatic brain injury. Medical Gas Research, 6(1), 3-9. [DOI:10.4103/20459912.179338] [PMID] [PMCID]

Li, Q., Han, X., \& Wang, J. (2016). Organotypic hippocampal slices as models for stroke and traumatic brain injury. Molecular Neurobiology, 53(6), 4226-37. [DOI:10.1007/s12035-015-9362-4] [PMID] [PMCID]

Loetscher, P. D., Rossaint, J., Rossaint, R., Weis, J., Fries, M., Fahlenkamp, A., et al. (2009). Argon: Neuroprotection in in vitro models of cerebral ischemia and traumatic brain injury. Critical Care, 13(6), R206. [DOI:10.1186/cc8214] [PMID] [PMCID]

Luft, A. R., \& Buitrago, M. M. (2005). Stages of motor skill learning. Molecular Neurobiology, 32(3), 205-16. [DOI:10.1385/ MN:32:3:205]
Macklis, J. D., \& Madison, R. D. (1990). Progressive incorporation of propidium iodide in cultured mouse neurons correlates with declining electrophysiological status: A fluorescence scale of membrane integrity. Journal of Neuroscience Methods, 31(1), 43-46. [DOI:10.1016/0165-0270(90)90007-3]

Miller, A. P., Shah, A. S., Aperi, B. V., Budde, M. D., Pintar, F. A., Tarima, S., et al. (2015). Effects of blast overpressure on neurons and glial cells in rat organotypic hippocampal slice cultures. Frontiers in Neurology, 6(20), 1-20. [DOI:10.3389/ fneur.2015.00020]

Millet, L. J., \& Gillette, M. U. (2012). Over a century of neuron culture: From the hanging drop to microfluidic devices. The Yale Journal of Biology and Medicine, 85(4), 501-21. [PMID] [PMCID]

Morrison III, B. Saatman, K. E., Meaney, D. F., \& Mcintosh, T. K. (1998). In vitro central nervous system models of mechanically induced trauma: A review. Journal of Neurotrauma, 15(11) 911-28. [DOI:10.1089/neu.1998.15.911] [PMID]

Morrison III, B., Cater, H. L., Benham, C. D., \& Sundstrom, L. E. (2006). An in vitro model of traumatic brain injury utilising two-dimensional stretch of organotypic hippocampal slice cultures. Journal of Neuroscience Methods, 150(2), 192-201. [DOI:10.1016/j.jneumeth.2005.06.014] [PMID]

Morrison, B., Cater, H. L., Wang, C. C., Thomas, F. C., Hung, C. T., Ateshian, G. A., \& Sundstrom, L. E. (2003). A tissue level tolerance criterion for living brain developed with an in vitro model of traumatic mechanical loading. Stapp Car Crash Journal, 47, 93-105. [DOI:10.4271/2003-22-0006] [PMID]

National Institutes of Health. (2016). Learn about clinical studies. Retrieved from https://clinicaltrials.gov/ct2/aboutstudies/learn\#WhoCanParticipate

Noraberg, J., Poulsen, F. R., Blaabjerg, M., Kristensen, B. W., Bonde, C., Montero, M., et al. (2005). Organotypic hippocampal slice cultures for studies of brain damage, neuroprotec tion and neurorepair. Current Drug Targets-CNS \& Neurological Disorders, 4(4), 435-52. [DOI:10.2174/1568007054546108] [PMID]

Roehl, A. B., Hein, M., Loetscher, P. D., Rossaint, J., Weis, J., Rossaint, R., et al. (2010). Neuroprotective properties of levosimendan in an in vitro model of traumatic brain injury. BMC Neurology, 10, 97. [DOI:10.1186/1471-2377-10-97] [PMID] [PMCID]

Rossaint, J., Rossaint, R., Weis, J., Fries, M., Rex, S., \& Coburn M. (2009). Propofol: Neuroprotection in an in vitro model of traumatic brain injury. Critical Care, 13, R61. [DOI:10.1186/ cc7795] [PMID] [PMCID]

Schoeler, M., Loetscher, P. D., Rossaint, R., Fahlenkamp, A V., Eberhardt, G., Rex, S., et al. (2012). Dexmedetomidine is neuroprotective in an in vitro model for traumatic brain injury. BMC Neurology, 12, 20. [DOI:10.1186/1471-2377-12-20] [PMID] [PMCID]

Smith, M., Piehler, T., Benjamin, R., Farizatto, K. L., Pait, M. C., Almeida, M. F., et al. (2016). Blast waves from detonated military explosive reduce GluR1 and synaptophysin levels in hippocampal slice cultures. Experimental Neurology, 286, 107-15. [DOI:10.1016/j.expneurol.2016.10.002] [PMID] [PMCID]

Stoppini, L., Buchs, P. A., \& Muller, D. (1991). A simple method for organotypic cultures of nervous tissue. Journal of Neuroscience Methods, 37(2), 173-82. [DOI:10.1016/0165-0270(91)90128-M] 
Vogel III, E. W., Effgen, G. B., Patel, T. P., Meaney, D. F., Bass, C. R. D., \& Morrison III, B. (2016). Isolated primary blast inhibits long-term potentiation in organotypic hippocampal slice cultures. Journal of Neurotrauma, 33(7), 652-61. [DOI:10.1089/ neu.2015.4045] [PMID] [PMCID]

Zhuang, L., Yang, T., Zhao, H., Fidalgo, A. R., Vizcaychipi, M. P., Sanders, R. D., et al. (2012). The protective profile of argon, helium, and xenon in a model of neonatal asphyxia in rats. Critical Care Medicine, 40(6), 1724-30. [DOI:10.1097/ CCM.0b013e3182452164] [PMID] 


\section{Supplementary information. 1}

\section{Organotypic Hippocampal Brain Slices (OHBS)}

The OHBS were obtained from 5-7-day old mice pups (C57BL/6N, Charles River Laboratories, Sulzfeld, Germany):

- The mice pups' heads were cut off with scissors. Their brains were rapidly removed and immersed in an ice-cold Preparation Medium (PM).

- Using a dissection microscope, the brains were then cut into two hemispheres.

- In the next step, the frontal brain and cerebellum were removed, and the hemispheres were cut into $400-\mu \mathrm{m}$ thick transverse slices with a McIllwain tissue chopper (The Mickle Laboratory Engineering Co. Ltd., UK).

- After further preparation was performed, the hippocampi slices were arranged onto the membrane of a MilliCell tissue culture insert (MilliCell-CM, Millipore Corporation, USA).

- Eventually, the inserts were placed in tissue culture plates. Growth Medium (GM) was placed underneath the tissue culture insert.

- The cell cultures were incubated for 14 days. The GM was changed 24 hours after preparation and after that every three days.

- For experiments, GM was exchanged by Experimental Medium (EM) containing propidium iodide (PI).

As described before (Grüßer et al., 2017), PM consists of 100\% Gey's balanced salt solution (Sigma-Aldrich, Munich, Germany) and $5 \mathrm{mg} / \mathrm{mL}$ D-(+)Glucose (Roth, Karlsruhe, Germany). GM consists of 50\% Eagle minimal essential medium with Earle's salts (Sigma-Aldrich, Munich, Germany), 25\% heat inactive horse serum (Sigma-Aldrich, Munich, Germany) and 25\% Hank's balanced salt solution (Sigma-Aldrich, Munich, Germany), $5 \mathrm{mg} / \mathrm{mL}$ D-(+)Glucose (Roth, Karlsruhe, Germany), HEPES buffer solution (Fluka, Bucks, Switzerland), 1 Vol.\% antibiotic/antimycotic solution: penicillin G GIBCO ${ }^{\text {TM }} 10000 \mathrm{U} / \mathrm{mL}$, streptomycin sulphate $10 \mathrm{mg} / \mathrm{mL}$, amphotericin B $25 \mu \mathrm{g} / \mathrm{mL}$ (Thermo Fisher Scientific Brand), L-glutamine (Sigma-Aldrich, Munich, Germany). EM consists of $75 \%$ Eagle minimal essential medium with Earle's salts (Sigma-Aldrich, Munich, Germany), 25\% Hank's balanced salt solution (Sigma-Aldrich, Munich, Germany), $5 \mathrm{mg} / \mathrm{mL}$ D-(+)glucose (Roth, Karlsruhe, Germany), HEPES buffer solution (Fluka, Bucks, Switzerland), 1 Vol.\% antibiotic/antimycotic solution: penicillin G GIBCO ${ }^{\mathrm{TM}} 10000 \mathrm{U} / \mathrm{mL}$, streptomycin sulphate $10 \mathrm{mg} / \mathrm{mL}$, amphotericin B $25 \mu \mathrm{g} / \mathrm{mL}$ (Thermo Fisher Scientific Brand), L-glutamine (Sigma-Aldrich, Munich, Germany) and $3 \mu \mathrm{L} /$ $\mathrm{mL}$ propidium iodide (PI) (Sigma Aldrich, Munich, Germany). 
This Page Intentionally Left Blank 